This item was submitted to Loughborough's Research Repository by the author.

Items in Figshare are protected by copyright, with all rights reserved, unless otherwise indicated.

\title{
Determining the effect of cricket leg guards on running performance
}

PLEASE CITE THE PUBLISHED VERSION

http://dx.doi.org/10.1080/02640414.2011.553962

PUBLISHER

() Routledge Taylor \& Francis

VERSION

AM (Accepted Manuscript)

LICENCE

CC BY-NC-ND 4.0

REPOSITORY RECORD

Webster, James, and Jonathan R. Roberts. 2019. "Determining the Effect of Cricket Leg Guards on Running Performance". figshare. https://hdl.handle.net/2134/11468. 
This item was submitted to Loughborough's Institutional Repository (https://dspace.lboro.ac.uk/) by the author and is made available under the following Creative Commons Licence conditions.

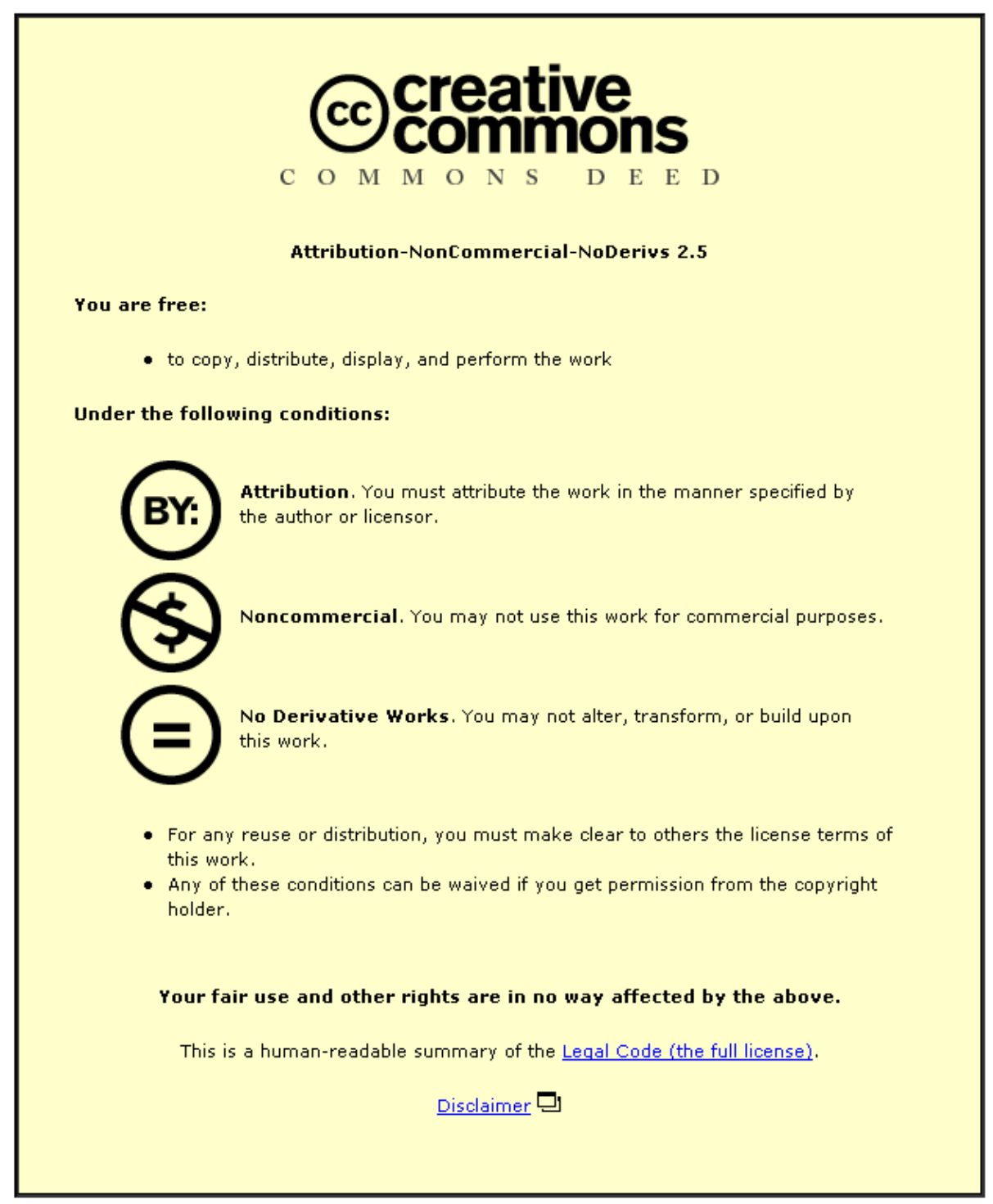

For the full text of this licence, please go to: http://creativecommons.org/licenses/by-nc-nd/2.5/ 


\title{
Determining the effect of cricket leg guards on running performance
}

\author{
JAMES WEBSTER \& JONATHAN ROBERTS
}

\author{
Sports Technology Institute, Loughborough University, Loughborough, Leicestershire, LE11 3TU, UK \\ Keywords: Cricket, Kinematics, Ground Reaction Force, Running Times
}

\begin{abstract}
Modern day cricket has experienced a shift towards limited over games, where the emphasis is on scoring runs at a rapid rate. The use of protective equipment in cricket is mandatory, however, players perceive that leg guards, in particular, can restrict their motion. The aim of this study was to determine the influence of cricket leg guards on running performance. Initial testing, revealed that wearing pads significantly increased the total time taken to complete three runs by up to $0.5 \mathrm{~s}$ when compared to running without pads $(\mathrm{p}<0.05)$. In addition, it was found that the degree of impedance was dependent on pad design and could not be solely attributed to additional weight. To assess possible causes of reduced running performance, a biomechanical analysis was performed, investigating running kinematics, stride parameters and ground reaction forces. The results revealed that the widest pad had the greatest effect on running kinematics, increasing hip abduction and decreasing hip extension resulting in a shortened stride length (by $0.10 \mathrm{~m}$ ) and increased stride width (by $0.12 \mathrm{~m}$ ) when compared to running without pads. Wearing pads also significantly increased peak braking force (by up to $0.3 \mathrm{BW}$ ), braking impulse (by up to $0.012 \mathrm{BW} . \mathrm{s}$ ), peak mediolateral force (by up to $0.17 \mathrm{BW}$ ) and mediolateral impulse (by up to 0.016 BW.s) when compared to running without pads, which resulted in reduced force applied in the direction of locomotion. The consequence of this reduction in running performance is an increased risk of being run-out or a reduction in the number of runs that could be scored from a particular shot.
\end{abstract}

\section{Introduction}

Cricket is an ever growing sport with the English Cricket Board estimating that participation in cricket increased by $24 \%, 27 \%$ and $15 \%$ in 2007, 2008 and 2009 respectively in England and Wales alone (ECB, 2009). This increased participation has led to a greater demand for equipment and with this has come greater competition between brands, striving to develop bats, helmets and protective clothing that out-perform their competitors' products. The perceived fit of cricket leg guards has been found to be a major factor affecting consumer satisfaction and, related to this is the effect of leg guards on running performance (Webster and Roberts, 2009).

Previous research in the wider field of personal protective equipment (PPE) has stated that the fit of a garment is crucial to the effectiveness and comfort of the equipment (Ashdown and DeLong, 1995). As a result, poor fit can affect performance due to restricted movement, increased heat strain and increased risk of injury as a result of exposure to hazards (Watkins, 1995; Milenković et al., 1999). Numerous studies have concluded that PPE can have detrimental effects on the range of motion of users, resulting in a loss of dexterity and mobility, thus affecting the ability to perform desired movements and tasks (Sheridan, 1954; Saul and Jaffe, 1955; Parssons and Egerton, 1985; Tremblay, 1989; Bellingar and Slocum, 1993). Research has identified the need to analyse the effect of fit on users' abilities to perform required movements in order to maximise performance, without sacrificing comfort or protection (Bellingar and Slocum, 1993; Adams and Keyserling, 1996; Huck et al., 1997).

Research within PPE has linked reduced mobility to material thickness, weight, protection level and design (Bellingar and Slocum, 1993; Watkins, 1995; Huck and Kim, 1997). Huck et al. (1997) found that altering a garment's fit in one area can result in the restriction of other movements. This was demonstrated by adding an elasticated waist at the back of protective overalls, which increased trunk flexion, but by doing this, knee and shoulder flexion decreased. These results demonstrate the need to understand the nature of movements performed whilst wearing the protective equipment and identify the design features that may restrict the user in order to maximise mobility. Suitable methods need to be developed which can be used to assess the motion of the user whilst performing typical movements and tasks.

There has been a substantial amount of research regarding the fit of a garment and its influence on movement. This research has historically based its findings on simplified movements, or maximal flexion and extension tests which only assess a user's ability to conduct routine movements whilst wearing the PPE in question, rather than testing realistic movements (Huck and Kim, 1997). The use of these quasi-static generic tests for specific items of sports PPE is questionable and warrants the development of a specific test protocol, enabling the testing of sports equipment in a more 
representative dynamic manner. Within sports PPE, the need for testing to be conducted whilst performing realistic movements has been identified in work by Green et al. (2000), who focused on assessing PPE whilst being used to perform required sporting motions. Green et al. (2000) assessed knee braces and their effect on typical football training drills (40 yard sprint time and time taken to complete a four cone agility test), and found that different knee braces affect performance to varying degrees.

Loock et al. (2006) measured the effect of cricket leg guards on running and turning times, concluding that the three sets of cricket leg guards they assessed did not significantly affect running or turning performance. The method utilised by Loock et al. (2006) allowed the effect of different leg guards on running speeds to be assessed in a realistic manner, increasing the validity of results when compared to other methods of assessment. The study, however, did not compare running in leg guards to running without pads. Also, since this study was conducted, the cricket market has seen the introduction of new lightweight modern pads which players have reported to be less restrictive than traditional pads (Webster and Roberts, 2009); further investigation was, therefore, warranted.

The aim of this study was to assess the influence of batting pads on straight-line running and turning performance and to identify design aspects that are responsible for any deterioration in performance. To achieve this, a two-stage methodology was adopted. The first stage focused on further developing previous work (Loock et al., 2006) by assessing the effect of different cricket leg guards on running speed especially when compared to running without leg guards. The second stage built on the results of the first stage of testing, through the use of biomechanical analysis techniques to investigate possible causes of reduced running speed.

\section{Stage 1: Running Time Analysis}

\section{Method}

Ten male cricketers, with a mean age of 19 years $( \pm 0.8$ years), all playing at county first or second team level, participated in a running time study. Each participant completed three consecutive runs whilst carrying a bat, starting with their lead foot on the crease. This was repeated four times with a one minute rest period between each set of three runs. The sequence of four sets of three runs was repeated for five different conditions (no pads, Pad 1, Pad 2, Pad 3 and a weighted comparison). The three pads tested within this study (Figure 1) were chosen as a suitable representation of the current market, with Pad $2\left(\mathrm{P}_{2}\right)$ representing a modern pad design, constructed of a single piece of moulded closed cell polyethylene foam. Pad $3\left(\mathrm{P}_{3}\right)$ represented a more traditional pad construction, comprising of multiple foams and pieces of cane for added support. Pad $1(\mathrm{Pl})$ was included as a compromise between the two extremes, and again comprised of multiple foams. As well as varying in construction, the pads also varied in mass, with $\mathrm{P}_{1}, \mathrm{P}_{2}$ and $\mathrm{P}_{3}$ having individual pad masses of $0.85 \mathrm{~kg}$, $0.5 \mathrm{~kg}$ and 0.9 $\mathrm{kg}$ per pad respectively. A no pad (NP) condition was included to determine an unrestricted benchmark against which the different pads could be compared. In order to determine if any impedance in running performance was solely attributable to additional mass, a "weighted comparison" (WC) was conducted. A $0.9 \mathrm{~kg}$ mass was positioned on the shin of each leg and strapped in place using elasticated straps. The mass was selected to be comparable with the heaviest pad used $\left(\mathrm{P}_{3}\right)$, but imposed minimal restriction around the knee and ankle, and had much less bulk (Figure 2).

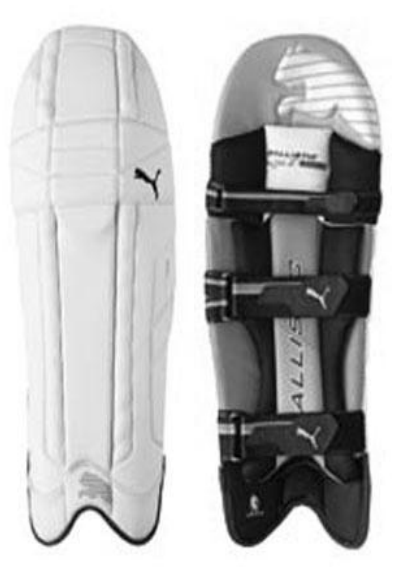

(P1)

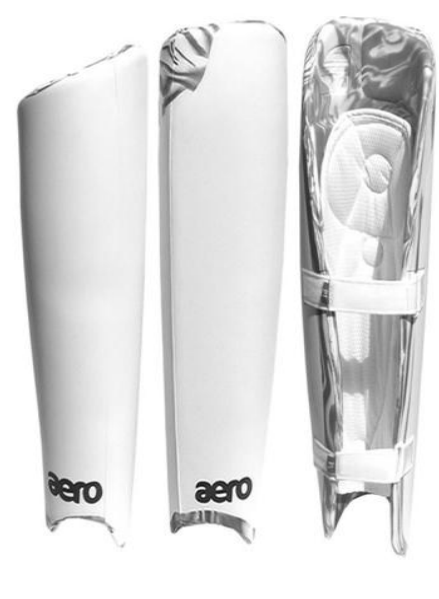

(P2)

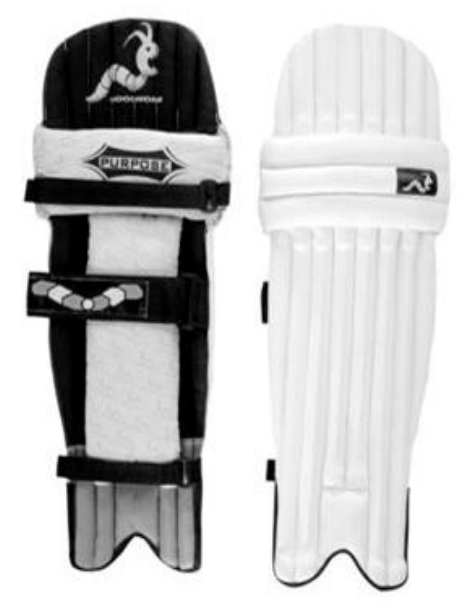

(P3)

Figure 1. Range of cricket leg guards used throughout the testing 
Running performance was evaluated using four Smart Speed light gates (Fusion Sport) positioned at each crease and at 5 meters in from each crease, as shown in Figure 3. Each light gate was set at shoulder height so that the player's torso would break the beam rather than their hand/arm or bat. The two light gates positioned on each crease were used to measure the "total-time" taken to complete 3 runs and the two gates positioned 5 meters before each crease were used to measure "turn-time", the time taken to run from 5 meters before the crease to 5 meters after. Each player was given a 15 minute rest between conditions to minimise any effects of fatigue, and condition order was randomised to prevent order effects. All methods used were approved by the Loughborough University Ethics Committee prior to subject recruitment.

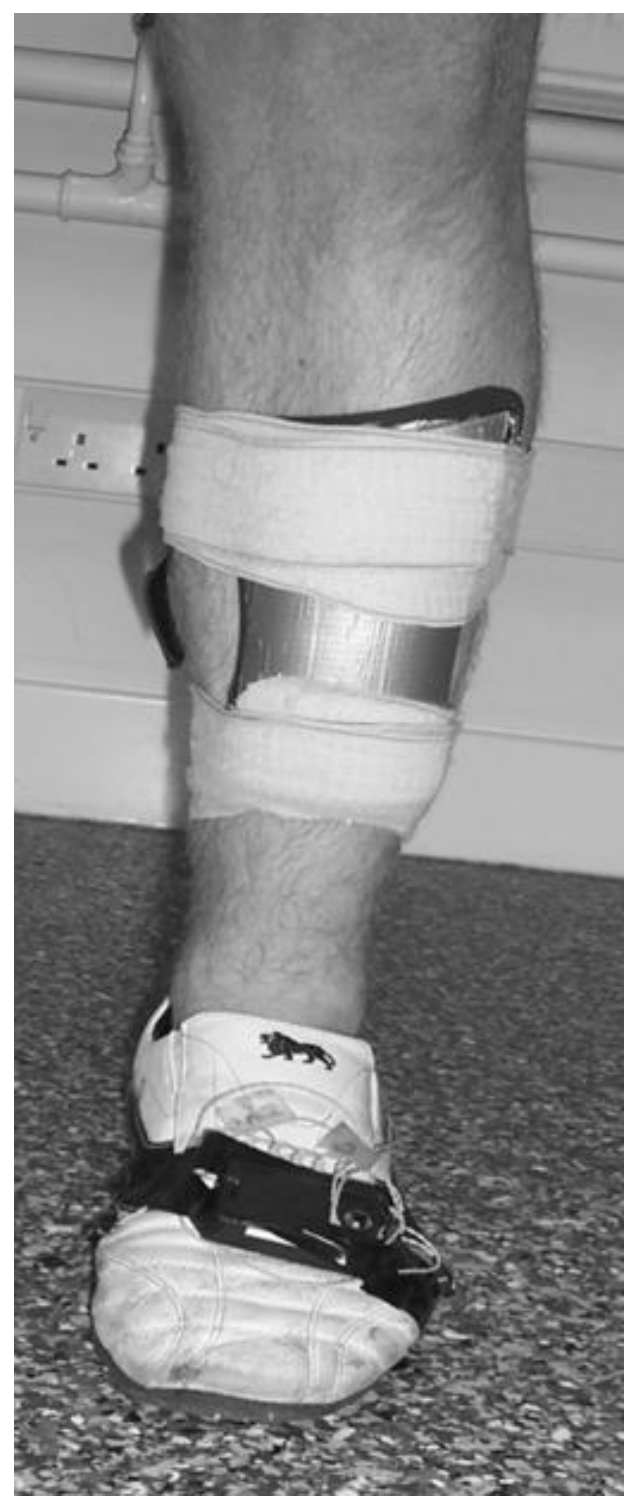

Figure 2. Positioning of the o.9kg mass for the WC tests

\section{Statistical analysis}

A Shapiro-Wilk test was used to establish the normality of data sets. All data presented in the results section was found to be normally distributed $(P>0.05)$, and so a oneway repeated measures ANOVA was used to determine if there was a main effect between conditions, and a TukeyKramer post-hoc test was completed to determine which results were significantly different $(P \leq 0.05)$. A Gabriel comparison interval was used to illustrate any significant differences identified between conditions (Gabriel, 1978). The Gabriel comparison was calculated by taking the standard error of the mean for a group and multiplying it by the studentized maximum modulus, this was then multiplied by the square root of one-half. The Gabriel comparison interval was then used to illustrate the upper and lower limits for $P=0.05$, with no overlap of intervals indicating a significant difference between the groups.

\section{Results}

The mean "total-time" for three runs was calculated individually for every participant for each condition, and a consistent trend was found across all participants, as illustrated in Figure 4. A significant difference was found between conditions $(F=10.34, P<0.05)$ in terms of "totaltime" and it can be seen that all the cricketers produced their fastest times when not wearing pads or an additional mass. The post-hoc results revealed that the "total-time" for all three padded conditions and the WC were significantly slower than the NP condition $(P<0.05)$ (Figure 5a). Mean "total-time" increased by up to 0.5 seconds when comparing $\mathrm{P}_{3}$ with the NP condition $(P<0.05)$ which translates to a distance of approximately 3 meters when running at a typical velocity of $6 \mathrm{~m} / \mathrm{s}$. Differences between padded conditions were also identified with $\mathrm{P}_{3}$ resulting in significantly slower times in comparison to $\mathrm{P}_{1}, \mathrm{P}_{2}$ and the WC $(P<0.05)$.

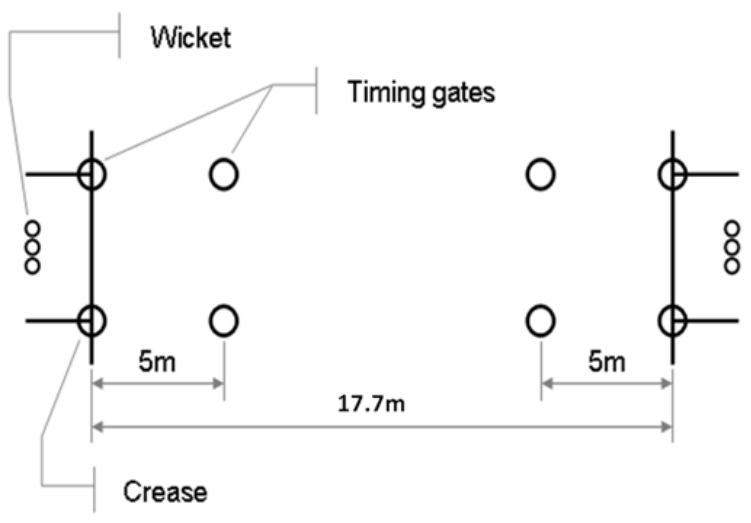

Figure 3. Schematic diagram of test set-up to measure running and turning times 


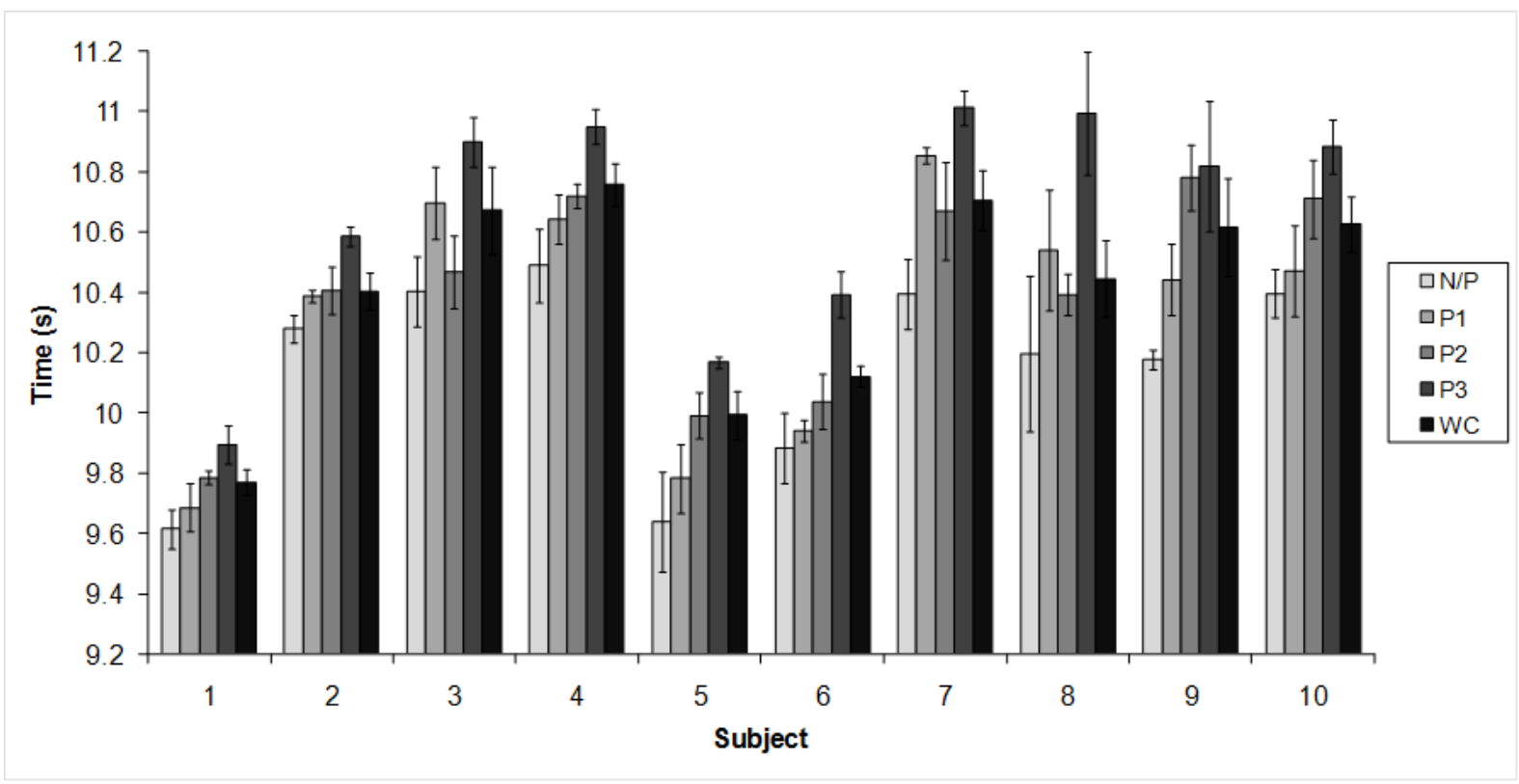

Figure 4. Mean "total time" for each condition for all 10 subjects ( \pm 1 standard deviation) Conditions: N/P = no pad, $\mathrm{P}_{1}=\mathrm{Pad} 1, \mathrm{P}_{2}=\mathrm{Pad} 2, \mathrm{P}_{3}=\mathrm{Pad}_{3}, \mathrm{WC}=$ weighted comparison

As well as "total-time", "turn-time" was measured; this was the mean time taken for turn one and turn two, as there was no significant difference found between turns within a trial $(P>0.05)$. No significant differences between conditions emerged from the statistical analysis $(P>0.05)$ (Figure $5 \mathrm{~b}$ ), suggesting that differences in time were due to the effect of pads on straight line running rather than on time taken to change direction.

These results suggest that running performance is inhibited by cricket pads, which decrease straight line running velocity. The addition of mass to the leg is clearly an important factor as "total-time" was greater for all conditions when compared to NP $(P<0.05)$. The increase in "total-time", however, was not directly proportional to the mass applied. There were no significant differences between $\mathrm{P}_{1}, \mathrm{P}_{2}$ or the WC $(P>0.05)$, despite a difference of up to $0.4 \mathrm{~kg}$ in mass whilst, $\mathrm{P}_{3}$ was found to inhibit performance to a greater degree than the WC $(P<0.05)$ despite being of equal mass. These results suggest that the decrease in performance is more complex, and so a further study was conducted to investigate the effect of leg guards on running biomechanics.
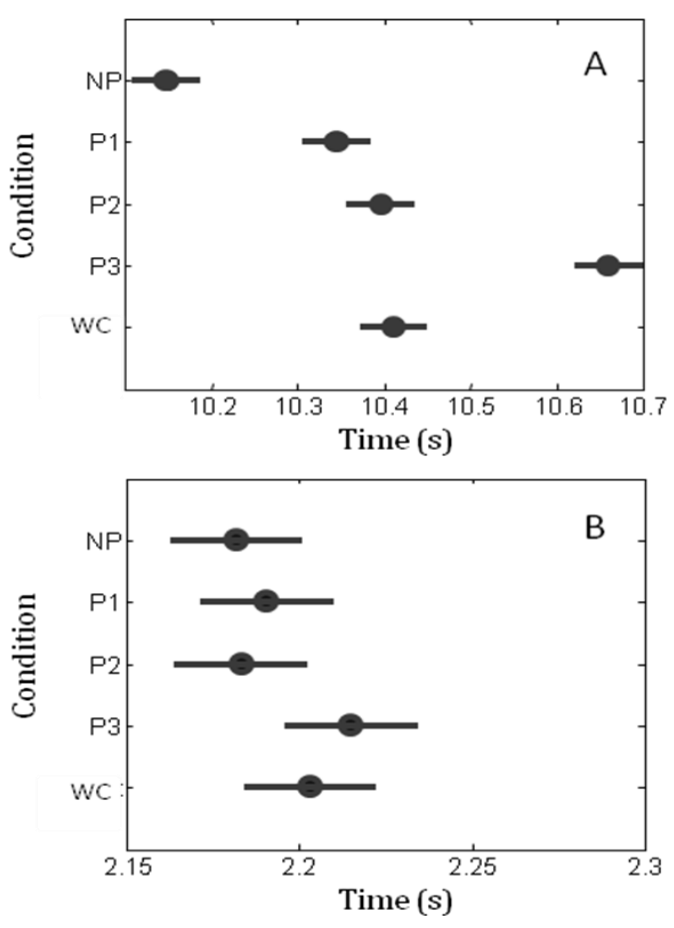

Figure 5. Mean \pm one Gabriel comparison interval for A)"total-time", B)"turn-time" for the five conditions. A significant difference is evident when the Gabriel comparison intervals do not overlap. Conditions: $\mathrm{N} / \mathrm{P}=$ no pad, $\mathrm{P} 1=$ Pad 1. $\mathrm{P}_{2}=\mathrm{Pad} 2, \mathrm{P}_{3}=\mathrm{Pad}$ 3, $\mathrm{WC}=$ weighted comparison. 


\section{Stage 2: Running Biomechanical Analysis}

\section{Method}

The aim of the second stage of testing was to identify if wearing different pads causes a measurable difference in running kinematics and kinetics, which could account for the reduction in running performance. For this study, nine male county ist and 2 nd team players were used with a mean age of 20.2 years ( \pm 1.1 years). The nine subjects within this testing were different to those used within Stage 1; this was deemed acceptable due to the consistent trend across the 10 subjects within Stage 1, demonstrated in Figure 4. Players were asked to perform six single runs for each of the five conditions outlined previously (NP, $\mathrm{P} 1$, $\mathrm{P}_{2}, \mathrm{P}_{3}$ and $\mathrm{WC}$ ); again, condition order was randomised. Single runs were considered, rather than three consecutive runs, as the focus of this study was on the section of a run where the player was at maximal speed rather than accelerating/ decelerating. This was the running phase, identified in Stage 1, where cricket pads impede performance. To replicate the running pattern, in terms of accelerations and decelerations, the players were asked to start from a stationary position and stop at a point $17.7 \mathrm{~m}$ from the start point. The start point was approximately $8 \mathrm{~m}$ from a force plate such that the player would strike the force plate within their typical stride pattern. The exact start position was determined from trial runs and was adjusted for each condition. Three of the six runs were designed to obtain a left foot strike, the other three a right foot strike, by varying the lead foot at the start point. For all data captures, the direction of locomotion was along the $\mathrm{x}$ axis of the force plate.

Motion data was collected using a four camera CXı Coda system sampling at $200 \mathrm{~Hz}$ with two integrated Kistler $9281 \mathrm{CA}$ force plates, sampling at $1000 \mathrm{~Hz}$. The kinematic data was taken from the middle segment of the run between $6 \mathrm{~m}$ and $10 \mathrm{~m}$ from the start point, with the force plate in the centre of this volume. Due to the protective equipment, a standard marker set up was not appropriate, as markers placed on the standard anatomical land marks at the knee and ankle would be covered by the leg guards. Therefore, the CAST technique was used (Cappozzo et al., 2005). For the static trial, the marker positions were captured whilst the participant was standing in the centre of the capture volume with their arms raised so no markers were occluded; a redundant marker set of 32 active markers was used (Figure 6), which incorporated additional markers on both thighs, shanks and feet for the clusters (Appendix 1). The static trial was an essential procedure within the data collection process and was used to determine the relative position of the cluster markers to those placed on the anatomical landmarks, which are deemed as being representative of the joint centres. Once the static trial had been captured, two walking and running trials were completed again without leg guards to assess accuracy of the marker set up. The joint centre positions and joint angles at the knee and ankle, when calculated using the clusters, were compared to those obtained using the markers on the
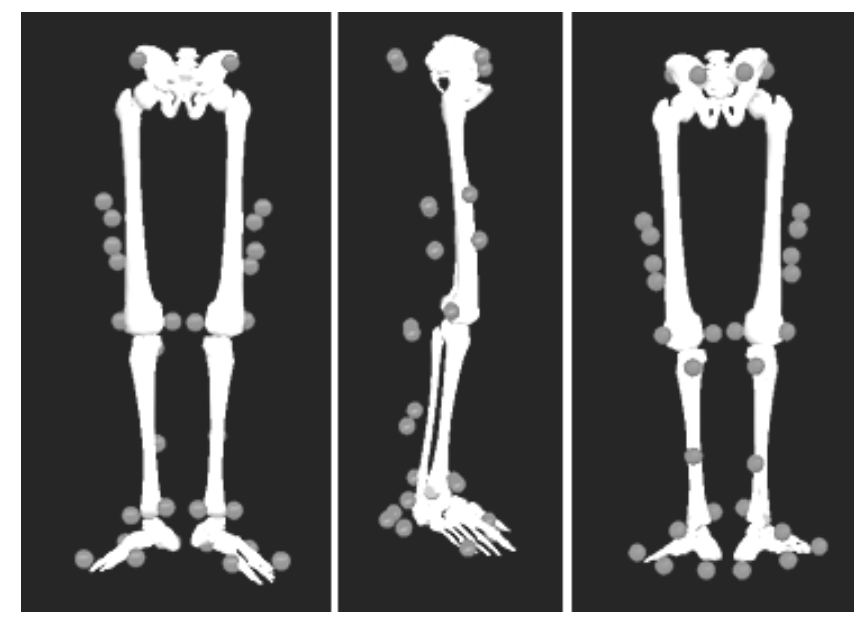

Figure 6. Static capture of full marker set up in Visual 3d (Version 4)

knee and ankle. A maximum root mean square difference in joint centre position of $2.45 \mathrm{~mm}$ and $1.97 \mathrm{~mm}$ was found across all subjects for the knee joint centre (KJC) and ankle joint centre (AJC) respectively. In addition, the maximum difference in knee angle between the cluster and anatomical marker placements was $4 \cdot 7^{\circ}$, which is less than the amount of skin artefact identified by Reinschmidt et al. (1997), therefore, the CAST method was deemed appropriate. Once the static trial and accuracy tests were completed, markers that would be covered by the leg guards were removed; these included the medial and lateral knee and ankle markers for both legs. Once all the data had been captured, a $3 \mathrm{D}$ kinematic model was built using Visual 3D software (Version 4) with the thigh cluster and foot cluster used to track movement of the knee and ankle joint centres. All data was filtered with a low-pass Butterworth filter with a cut off frequency of $12 \mathrm{~Hz}$ in accordance with Hunter et al. (2005).

The measurements taken from this study were kinematic parameters (hip, knee and ankle joint angles and velocities (Cardan angles, $x y z$ ), stride width, stride length, stance time, stride frequency) along with ground reaction forces (GRF) $(x, y, z)$. Stride length was calculated as the distance between proximal end position of the foot at ipsilateral heel strike to the proximal end position of the foot at the next ipsilateral heel strike. The vector between these two points was then used in the calculation of stride width. The cross product of this vector with the position of the intermediate step gave the stride width i.e. the medio-lateral distance between proximal end position of the foot at ipsilateral heel strike to the proximal end position of the foot at the next contralateral heel strike.

The GRF data was normalised to body weight (BW) by dividing the force data by each participant's weight for the NP condition and dividing by their weight plus the weight of the pad or leg weight for the other conditions. This was to compensate for differences in subject and pad weight. The anterior/posterior, mediolateral and vertical Journal of Sports Sciences, 2011; 1-12 
impulses were also considered as they reflect the change in velocity of the center of mass during the respective periods and in the respective directions. The area under the force-time curve was used to calculate impulse in each of the three axes and was normalised to body weight as described above; overall impulse was calculated as propulsive impulse less braking impulse in the anterior/posterior direction (Hunter et al., 2005).

Kinanthropometric data was collected to analyse the fit of the pad, enabling a comparison between fit and performance to be made. The measurements taken were circumference and width of the leg without pads at the thigh ( $8 \mathrm{~cm}$ above the top of the patella), knee (across the point of articulation) and calf (mid point between the knee and ankle). These measurements were also taken in each of the three padded conditions to give the combined width and circumference of the leg and pad.

\section{Statistical analysis}

Initially, a cross-correlation was performed on the hip, knee and ankle data to determine if movement patterns were consistent between trials and between subjects, with a value of o representing no correlation and a value of 1 representing a strong correlation. As with the running time data in Stage 1, a Shapiro-wilks test was used to determine if the data was normally distributed. Again, all data presented in the results section was found to be normally distributed $(P>0.05)$ and so a one-way repeated measures ANOVA with a post-hoc Tukey-Kramer test was completed to determine which results were significantly different $(P \leq 0.05)$. A Gabriel comparison interval was used to illustrate significant differences between conditions (Gabriel, 1978).

\section{Results}

\section{Ground Reaction Force}

Significant differences in GRF were found between conditions (Table 1). The GRF data for the anterior/posterior $(x)$ direction is shown in Figure 7 , which illustrates the differences in maximum force and impulse between conditions. For all metrics, apart from maximum propulsive force, a similar trend can be seen in the data with significant differences $(P<0.05)$ between the NP condition, $\mathrm{P}_{3}$ and a group containing $\mathrm{P}_{1}, \mathrm{P}_{2}$ and the WC. The NP condition resulted in the smallest braking force and braking impulse which, combined with the largest propulsive impulse, resulted in the greatest overall impulse. At the other extreme, the smallest overall impulse was for $\mathrm{P}_{3}$, which was a consequence of a large braking impulse and braking force and a smaller propulsive impulse. Occupying the middle ground between these two extremes were $\mathrm{P}_{1}, \mathrm{P}_{2}$ and the WC, amongst which there were no significant differences. This trend is the same as that seen in Figure 5a, which, perhaps not surprisingly, indicates a strong correlation between "total-time" and anterior/posterior GRF.

In terms of mediolateral forces, a similar trend can also be seen, certainly between NP and the three padded conditions, as illustrated in Figure 8. The WC condition, however, now differs significantly from $\mathrm{P}_{1}$ and $\mathrm{P}_{2}$ $(P<0.05)$, resulting in mediolateral forces similar to the NP condition. No significant differences were found between conditions in the vertical force data (Figure 8).

\section{Table 1. Results of one-way ANOVA on GRF data}

\begin{tabular}{|l|l|l|l|l|}
\hline \multirow{2}{*}{$\begin{array}{l}\text { GRF } \\
\text { Component }\end{array}$} & \multicolumn{2}{|l|}{ Maximum force } & \multicolumn{2}{l|}{ Impulse } \\
\cline { 2 - 5 } & $F$ & $P$ & $F$ & $P$ \\
\hline $\begin{array}{l}\text { Posterior } \\
\text { (braking) }\end{array}$ & 16.35 & $<0.05$ & 72.57 & $<0.05$ \\
\hline $\begin{array}{l}\text { Anterior } \\
\text { (propulsive) }\end{array}$ & 0.63 & $>0.05$ & 9.38 & $<0.05$ \\
\hline Mediolateral & 15.67 & $<0.05$ & 11.94 & $<0.05$ \\
\hline Vertical & 2.15 & $>0.05$ & 0.80 & $>0.05$ \\
\hline
\end{tabular}

\section{Kinematic analysis}

The effect of cricket leg guards on running kinematics was assessed to determine if changes in GRF and effectively running velocity were a result of differences in running gait. To investigate this, hip, knee and ankle flexion/extension, hip abduction/ adduction and joint angular velocities were studied. Initially, a cross correlation was performed on the mean angular data between subjects and conditions. To determine the similarity of movement between subjects, a crosscorrelation was computed for every pair of subjects for a given condition and a particular joint angle. This was repeated for all other conditions and a mean coefficient calculated for that joint angle. Strong correlations (>0.811) emerged, suggesting that there is limited variation in movement pattern between subjects. A similar method was followed to study differences between conditions. A cross-correlation was computed for every pair of conditions for a given subject and a particular joint angle. This was repeated for all subjects and the mean coefficient calculated for each joint angle. Strong correlations (>0.863) again emerged, suggesting that there is considerable similarity for the joint angle data from all five conditions in terms of movement pattern, movement duration and degree of flexion/ extension and abduction/ adduction.

Figures 9 and 10 illustrate the left hip, knee and ankle kinematics for Subject 3. These results were deemed to be representative of all nine subjects' data, due to the high correlation between subjects. To determine if there were any significant differences in joint angles, the points of maximum flexion/extension at the hip, knee and ankle for each stride were considered along with peak angular velocities. No significant differences $(0.331 \leq F \leq 1.878$, $P>0.05$ ) were found at the knee or ankle (Figure 9), suggesting that changes in GRF and running times are not as a result of changes in maximum flexion and extension of the knee or ankle. 

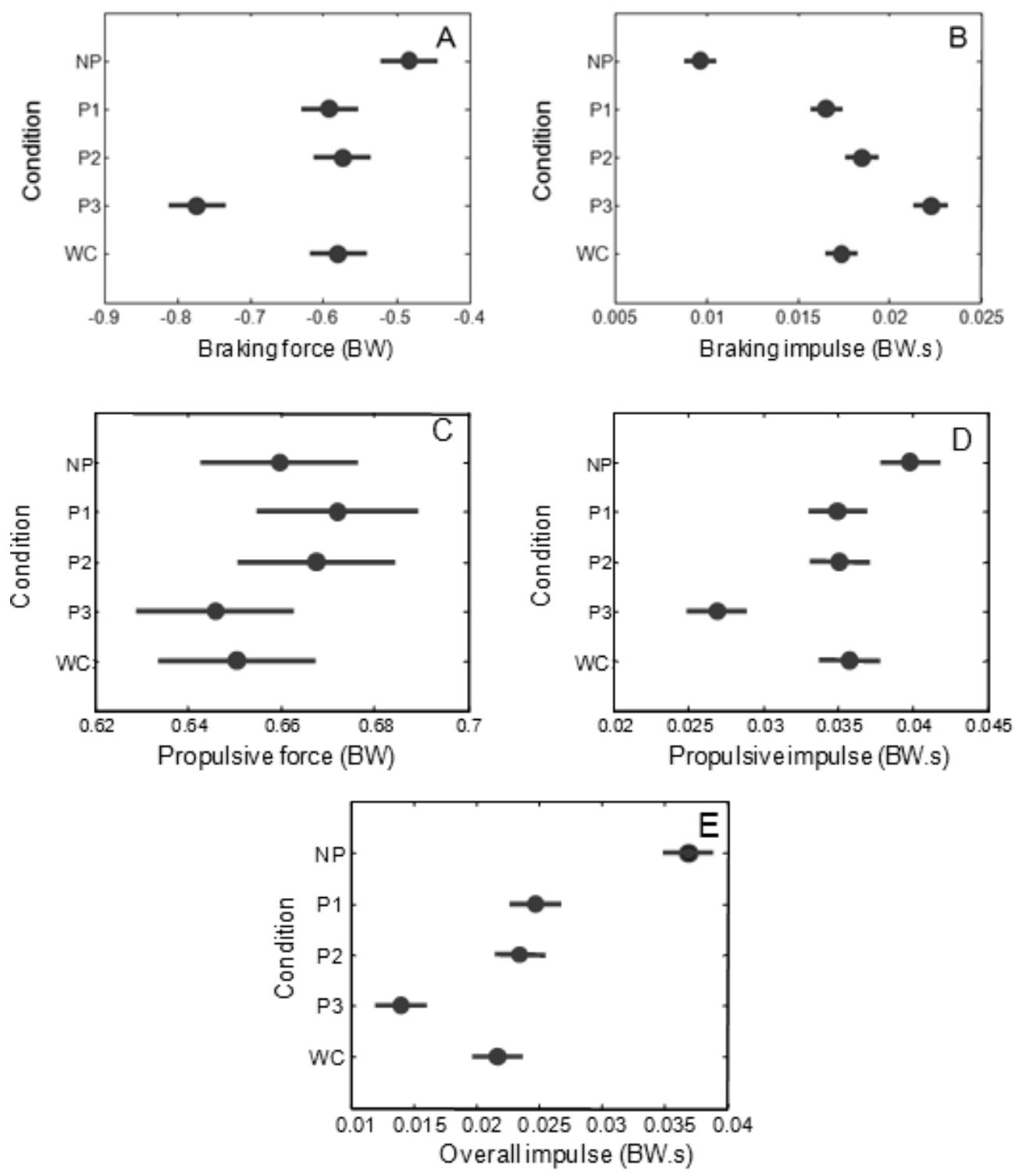

Figure 7. Mean GRF \pm one Gabriel comparison interval for all conditions in regards to A) maximum braking force, $\mathrm{B}$ ) braking impulse, C) maximum propulsive force, D) propulsive impulse, E) overall impulse. A significant difference is evident when the Gabriel comparison intervals do not overlap.

At the hip (Figure 10), no significant differences werefound between NP, WC, $\mathrm{P}_{1}$ or $\mathrm{P}_{2}$ in terms of either peak flexion/extension or peak adduction/abduction ( $>>0.05) . P_{3}$, however, was found to significantly decrease peak hip extension and increase peak abduction when compared to the other four conditions $(P<0.05)$. Compared to the NP condition, on average peak extension decreased by $9.7^{\circ}$ and peak abduction by $5.2^{\circ}$ when wearing $\mathrm{P}_{3}$; at the moment of left heel strike, the difference in abduction angle increased further to $5.4^{\circ}$. No corresponding differences were found in the peak angular velocity data about either axis of motion at the hip (o.693 $\leq F \leq 0.742, P>0.05$ ).

The kinematic data was also used to calculate specific stride parameters. $\mathrm{P}_{3}$ was found to significantly increase the stride width of players by $0.12 \mathrm{~m}(P<0.05)$ (Figurenb), and decrease stride length by $0.1 \mathrm{~m}(P<0.05)$ (Figure 11a) on average when compared to the NP condition. No significant differences were found in either stride width or stride length between NP, $\mathrm{P}_{1}, \mathrm{P}_{2}$, and the WC $(P>0.05)$, nor were any significant differences found for other stride parameters (Table 2) including step frequency $(F=0.419$, $P>0.05)$, step time $(F=0.237, P>0.05)$ and stance time $(F=0.927, P>0.05)$.

Finally, the subject's running velocity was calculated using the velocity of the pelvis. Significant differences in running velocity were found between conditions $(F=1.436$, $P<0.05)$ with the trend reflecting the differences in "total time" calculated in Stage 1. The fastest velocities were achieved in the NP condition, the slowest wearing $\mathrm{P}_{3}$, with the other conditions grouped together in between.

Journal of Sports Sciences, 2011; 1-12 

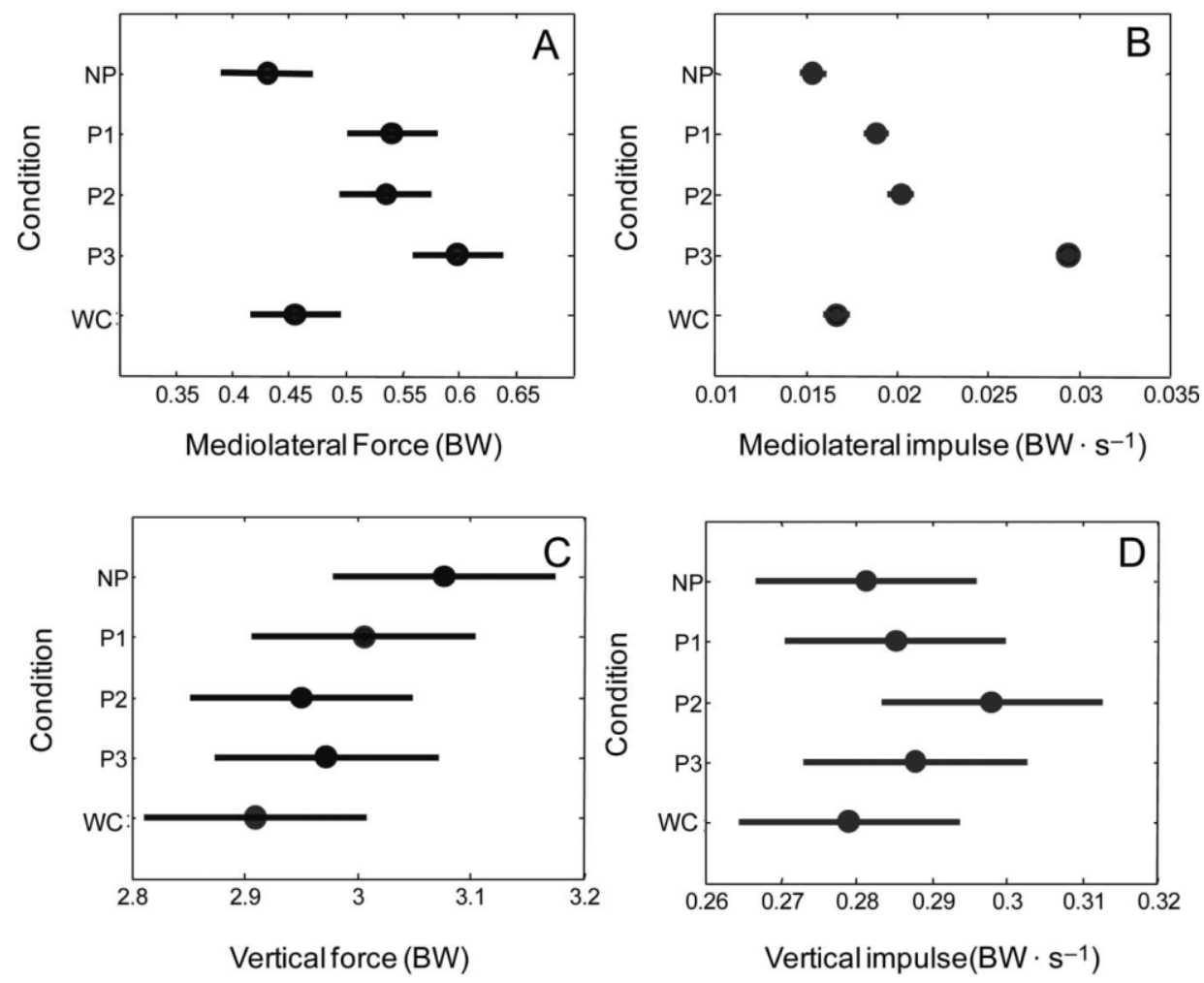

Figure 8. Mean GRF \pm one Gabriel comparison interval for all conditions in regards to A) maximum mediolateral force, B) medial lateral impulse, C) maximum vertical force and D) vertical impulse. A significant difference is evident when the Gabriel comparison intervals do not overlap.
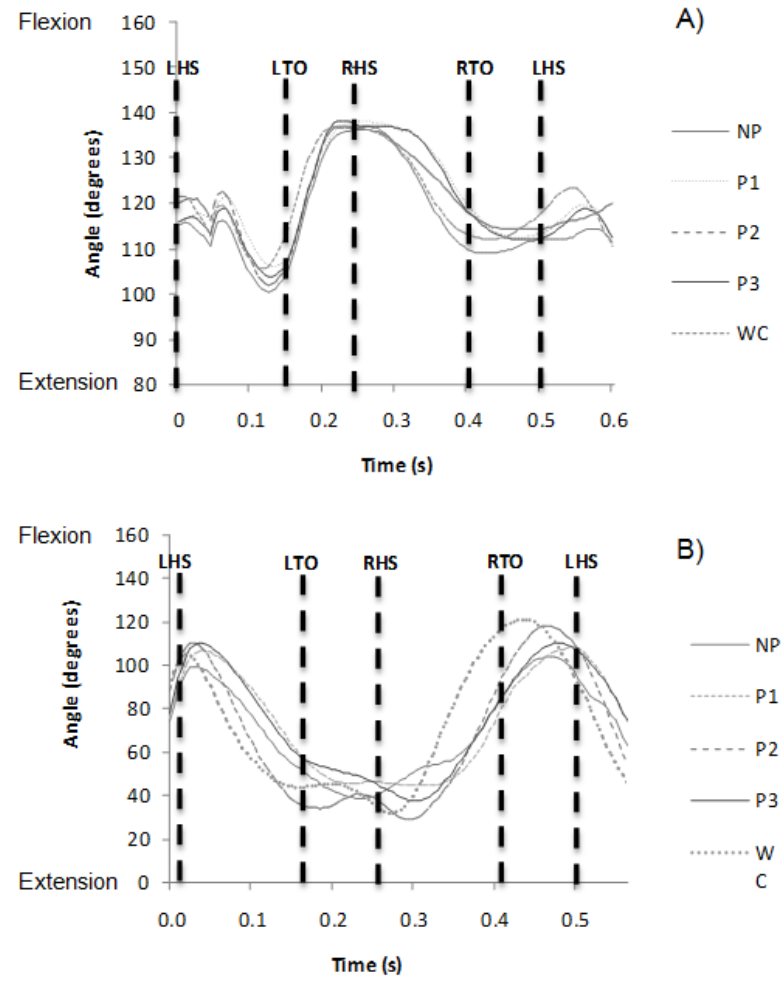

Figure 9. Mean joint kinematics for Subject 3 for one complete stride for A) left knee angle and B) left ankle angle for all five conditions (left heel strike (LHS), left toe off (LTO), right heel strike (RHS) and right toe off (RTO))
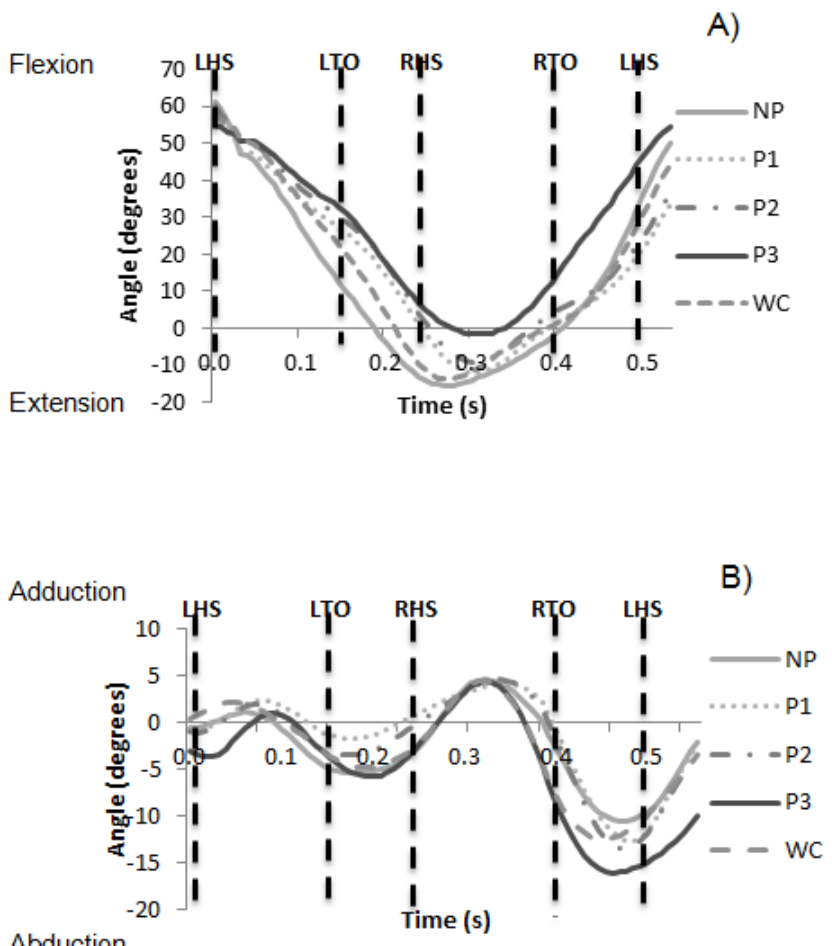

Figure 10. Mean joint kinematics for Subject 3 for one complete stride for A) left hip flexion/extension and B) left hip abduction/adduction for all five conditions (left heel strike (LHS), left toe off (LTO), right heel strike (RHS) and right toe off (RTO)) 


\section{Kinanthropometry}

Six static measurements were taken for each condition, including width and circumference of the thigh, knee and calf. The results are shown in Figure 12 and were used to determine if there was a relationship between pad size and impedance. It was found that all three pads added a significant amount of size to the legs in terms of width and circumference at all three locations. The biggest changes in dimension were around the knee and calf for all three pads in respect to width and circumference. Overall, $\mathrm{P}_{3}$ added the greatest amount of bulk, increasing the width at the knee by $10.1 \mathrm{~cm}$ compared to $7.4 \mathrm{~cm}$ and $8.5 \mathrm{~cm}$ on average for $\mathrm{P}_{1}$ and $\mathrm{P}_{2}$ respectively. These results suggest that the significant increase in stride width for $\mathrm{P}_{3}$ could be due to the added bulk in between the legs, preventing a natural running motion (as demonstrated in the NP condition) for $\mathrm{P}_{3}$ alone.
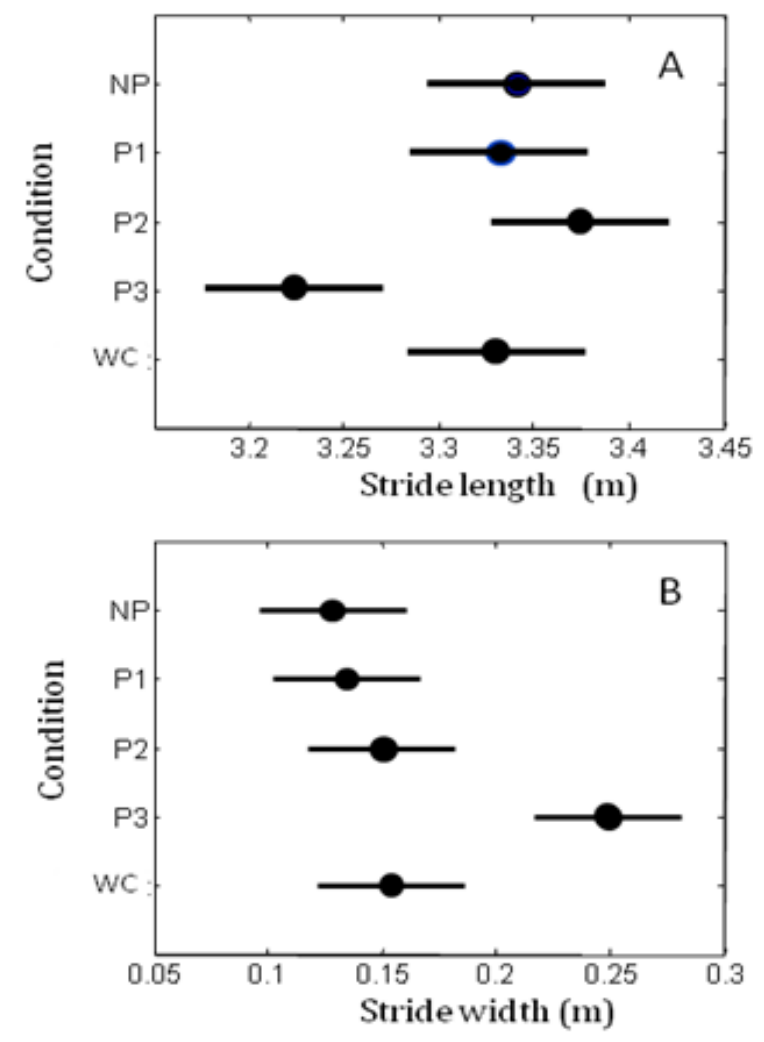

Figure 11. Mean \pm one Gabriel comparison interval for A) stride length and B) stride width for all five conditions. A significant difference is evident when the Gabriel comparison intervals do not overlap.

\section{Discussion}

From the first stage of testing, it can be seen that wearing cricket pads has a negative effect on straight-line running performance when compared to running without pads and, contrary to Loock et al.'s (2006) findings, the magnitude of this effect can vary between pads. Adding mass to the leg reduced running velocity and increased "total-time" taken to complete three runs although the differences between conditions were not directly proportional to the change in additional mass, suggesting other factors were also responsible.

The reason for the reduction in running velocity is evident in the GRF data. When compared to the NP condition, the other four conditions resulted in higher braking impulses, lower propulsive impulses and, therefore, lower overall impulses in the anterior/posterior direction i.e. the direction of travel. Wearing pads also increased mediolateral force which, along with the reduction in anterior/posterior force, suggests they also influence the direction of the resultant force applied. The largest mediolateral force occurred for $\mathrm{P}_{3}$, the widest pad, whilst no significant difference was evident between the NP and WC conditions implying that the magnitude of this effect is related to the size of the pad worn

Despite considerable differences in GRFs and, consequently, running velocities between conditions, few significant differences were observed in the kinematic data. $\mathrm{P}_{3}$ decreased peak hip extension and increased peak abduction which resulted in a greater stride width and a shorter stride length when compared to the other conditions. No significant differences emerged, however, between the NP condition and either $\mathrm{P}_{1}, \mathrm{P}_{2}$ or the WC condition. This suggests that either the greater accuracy and repeatability of the GRF data enabled more subtle differences to emerge or that adding mass to the leg does not affect running kinematics. Other studies have concluded that adding a $2 \mathrm{~kg}$ mass to each ankle affects stride length by less than 1\% (Martin, 1985; Royer et al., 2001; Browning et al., 2007) which implies that pads, such as $\mathrm{P}_{3}$, alter the running kinematics of the athlete because of their size. This is particularly apparent when the added size to the leg forces an increase in natural stride width. As an approximate guide, the size of the pad inside the leg needs to be minimised and kept below the width of the natural running stride as measured in the NP condition

Table 2. Kinematic data group + 1 standard deviation

\begin{tabular}{|l|l|l|l|l|l|}
\hline \multirow{2}{*}{ Stride Parameter } & \multicolumn{4}{l}{ Group Mean $( \pm 1 \mathrm{SD})$} \\
\cline { 2 - 6 } & $\mathrm{NP}$ & $\mathrm{P} 1$ & $\mathrm{P} 2$ & $\mathrm{P}_{3}$ & $\mathrm{WC}$ \\
\hline Step Frequency $(\mathrm{Hz})$ & $3.96 \pm 0.27$ & $3.89 \pm 0.26$ & $3.94 \pm 0.26$ & $3.87 \pm 0.28$ & $3.91 \pm 0.29$ \\
\hline Step time $(\mathrm{s})$ & $0.25 \pm 0.02$ & $0.26 \pm 0.02$ & $0.25 \pm 0.02$ & $0.26 \pm 0.016$ & $0.26 \pm 0.0$ \\
\hline Stance time $(\mathrm{s})$ & $0.17 \pm 0.014$ & $0.17 \pm 0.013$ & $0.16 \pm 0.053$ & $0.17 \pm 0.025$ & $0.17 \pm 0.013$ \\
\hline Velocity of Pelvis $(\mathrm{m} / \mathrm{s})$ & $6.35 \pm 0.15$ & $6.13 \pm 0.13$ & $6.14 \pm 0.18$ & $6.01 \pm 0.21$ & $6.14 \pm 0.20$ \\
\hline
\end{tabular}



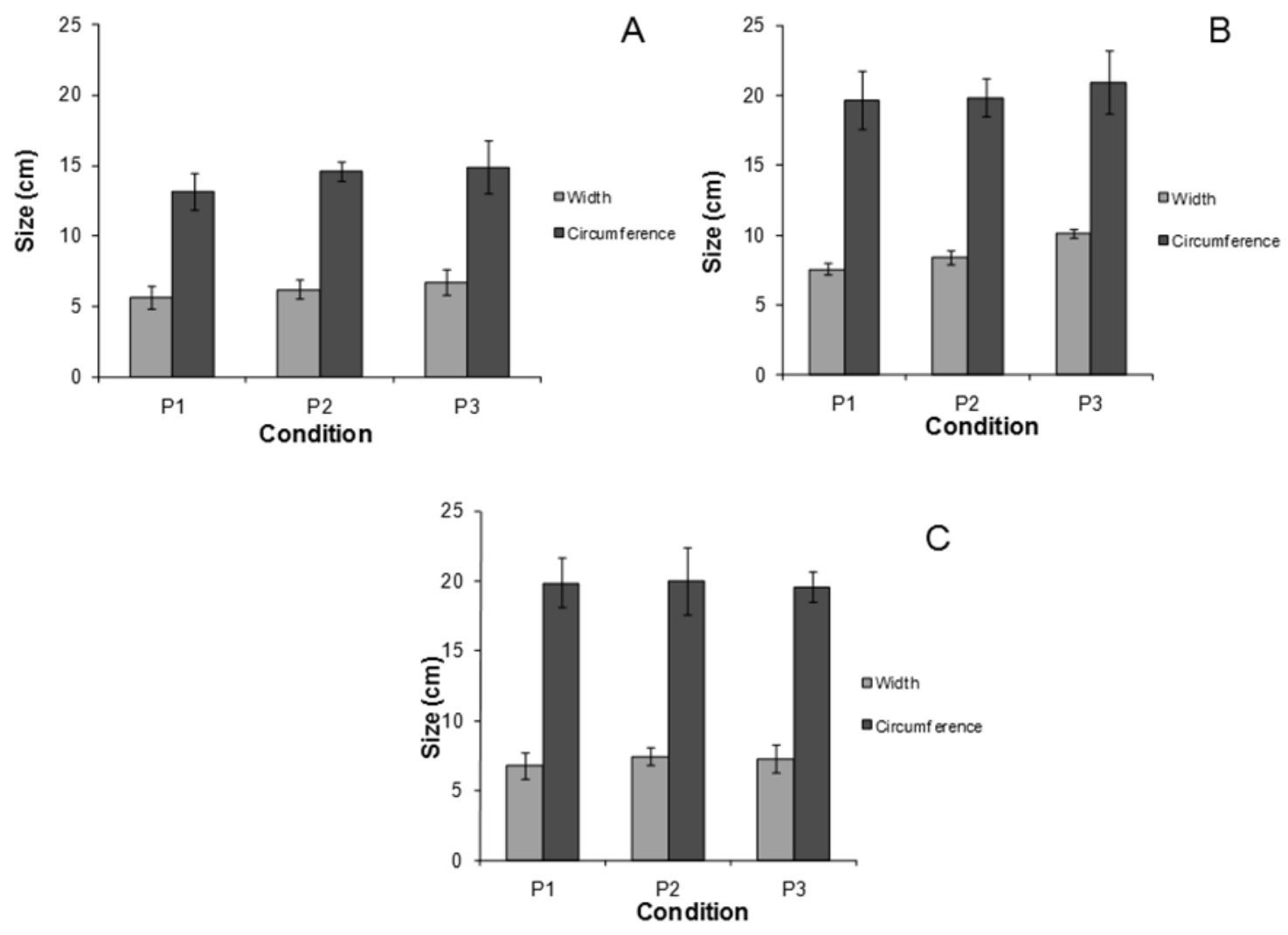

Figure 12. Additional leg width and circumference \pm 1 SD from wearing cricket pads at A) the thigh, B) the knee and $C$ ) the calf.

(approximately $10 \mathrm{~cm}$ ) in order to minimise changes in running gait, in particular hip extension/flexion and hip adduction/abduction. From these results, it is suggested that leg guards must not add more than $10 \mathrm{~cm}$ to the width of the leg, and in particular can not add more than $5 \mathrm{~cm}$ to the inside of the leg.

Although, the inclusion of the biomechanical analysis has helped to identify pad design features which could be detrimental to running performance, one limitation is the number of variables between pads. Therefore, in future, to assess which design features have the greatest effect on performance, several iterations of the same pad could be used, where one variable is altered on each version of the pad, allowing the effect of each variable (weight, size etc.) on performance to be determined.

\section{Conclusion}

This study has measured and quantified the influence of PPE in cricket on running performance. A test procedure was developed allowing a biomechanical analysis of routine movements typically performed within the sport. The results suggest that all pads significantly hinder running performance when compared to running without PPE. In addition, significant variations in the degree of restriction between pads were discovered, which could not be solely attributed to the additional mass of the pads. A biomechanical analysis revealed that wider pads, such as $P_{3}$, significantly decrease peak hip extension and stride length, increase hip abduction and stride width, resulting in a reduced overall impulse in the direction of travel and increased mediolateral forces. Consequently, running velocity decreases and the "total time" taken to complete three runs increases. Through the development of new pads with minimal restriction, whilst maintaining protective levels, running performance can be improved, resulting in either a decreased risk of being run out, or the opportunity to increase the number of runs scored from a shot, which would be particularly appealing in limited over matches. 


\section{REFERENCES}

Adams, P. and Keyserling, W. (1996). Performance of Protective Clothing: Fifth Volume, ASTM 1237, Philadelphia, PA: American Society for Testing and Materials.

Ashdown, S.P. and DeLong, M. (1995). Perception testing of apparel ease variation. Applied Ergonomics, 26(1), 47-54.

Bellingar, T.A. and Slocum, A.C. (1993). Effect of protective gloves on hand movement: an exploratory study. Applied Ergonomics, 24(4), 244-250.

Browning, R. C., Modica, J. R., Kram, R. and Goswami, A. (2007) The effects of adding mass to the legs on the energetics and biomechanics of walking. Medicine and Science in Sports and Exercise, 39(3), 515-525.

Cappozzo, A., Della Croce, U., Leardini, A. and Chiari, L. (2005) Human movement analysis using stereophotogrammetry. Part 1. Theoretical background. Gait Posture, 21, 186-96.

ECB (2009) "Twelth Man" http://www.ecb.co.uk/twelfthman/interactive/q-anda/2841,2841,QA.html

Gabriel, K. R., (1978). A simple method of multiple comparison of means. Journal of The American Statistical Association, 73, 724-729.

Green, D. L., Hamson, K. R., Bay, R. C. and Bryce, C. E. (200o). Effects of protective knee bracing on speed and agility. The American Journal of Sports Medicine, 28, (4), 453-459.

Huck, J. and Kim, Y. (1997). Coveralls for grass fire fighting. International Journal of Clothing Science and Technology, 9, 346-359.

Huck, J., Maganga, O. and Kim, Y. (1997). Protective overalls: Evaluation of garment design and fit. International Journal of Clothing Science and Technology, 9, 45-61.

Hunter J. P., Marshall, R. N., and McNair, P. J., (2005). Relationships between ground reaction force impulse and kinematics of sprint-running acceleration. Journal of Applied Biomechanics, 21(1), 31-43.

Loock, N., Du Toit D. E., Ventner, D. J., and Stretch, R. A. (2006). Effect of different types of cricket batting pads on the running and turning speed in cricket batting. Sports Biomechanics, 5(1) 15-22.

Martin, P. E. (1985). Mechanical and physiological responses to lower extremity loading during running. Medicine and Science in Sports and Exercise, 17, 427-433.

Milenković, L., Škundrić, P., Sokolović, R. and Nikolić, T. (1999). Comfort properties of defense protective clothings. Working and Living Environmental Protection, 1(4), 101-106

Parssons, K.C. and Egerton, D.W. (1985). The effect of glove design on manual dexterity in neutral and cold conditions. In: Contemporary Ergonomics (edited by D.J. Oborne), 203-209. London: Taylor and Francis.

Reinschmidt, C., van den Bogert, A.J., Nigg, B.M., Lundberg, A. and Murphy, N., (1997). Effect of skin movement on the analysis of skeletal knee joint motion during running. Journal of Biomechanics 30, 729-732.

Royer, T. D., and P. E. Martin (2001). Altered motor control strategies during the swing phase of walking with leg loads. Medicine and Science in Sports and Exercise, 33(5), 152-158.

Saul, E.V. and Jaffe, J. (1955). The effects of clothing on gross motor performance. USA: Quartemaster Research and Development Centre, US Army.

Sheridan, T.B. (1954). An experimental study of physical criteria for evaluating handcovering designs, Masters edn, University of California, USA.

Tremblay, J.F. (1989). Evaluation of functional fit and comfort of chemical protective gloves for agricultural workers, Masters Thesis, University of Alberta, Canada.
Watkins, S.M. (1977). The design of protective equipment for ice hockey. Home Economics Research Journal, 5(3), 164-168.

Watkins, S.M. (1995). Clothing: The portable environment. (2nd ed). USA: Iowa State University Press.

Webster, J. M. and Roberts, J. (2009). Incorporating subjective end-user perceptions in the design process: A study of leg guard comfort in cricket. Proc. IMECHE Part P: Journal of Sports Engineering and Technology, 223, 49-62.

Wheat, K.L. and Dickson, M.A. (1999). Uniforms for collegiate female golfers: Cause for dissatisfaction and role conflict? Clothing and Textiles Research Journal, 17, 1-10. 
Appendix 1

\begin{tabular}{|c|c|c|}
\hline Marker & Definition & Position \\
\hline L/RASI & Left anterior super iliac & \multirow[t]{2}{*}{ Bony protrusion of the anterior superior iliac. } \\
\hline RASI & Right anterior super iliac & \\
\hline LPSI & Left posterior super iliac & \multirow[t]{2}{*}{ Dimples created by the posterior super iliac. } \\
\hline RPSI & Right posterior super iliac & \\
\hline LTHAS & Left thigh anterior superior & \multirow[t]{2}{*}{ Placed on the left and right thigh } \\
\hline RTHAS & Right thigh anterior superior & \\
\hline LTHPS & Left thigh posterior superior & \multirow{2}{*}{$\begin{array}{l}\text { Placed on the thigh approximately } 2 \text { inches } \\
\text { posteriorly to the LTHAS or RTHAS. }\end{array}$} \\
\hline RTHPS & Right thigh superior & \\
\hline LTHAI & Left thigh anterior inferior & \multirow{2}{*}{$\begin{array}{l}\text { Placed on the thigh, } 1 \text { inch below the L/ RTHAS } \\
\text { and } 2 \text { inches forward of the line between L/ } \\
\text { RTHIS and L/ RTHII. }\end{array}$} \\
\hline RTHAI & Right thigh anterior inferior & \\
\hline LTHPI & Left thigh posterior inferior & \multirow{2}{*}{$\begin{array}{l}\text { Placed on the thigh, } 1 \text { inch below the L/ RTHPS } \\
\text { and } 2 \text { inches behind the L/ RTHAI and L/ } \\
\text { RTHAI. }\end{array}$} \\
\hline RTHPI & Right thigh posterior inferior & \\
\hline LKNEL & Left knee Lateral & \multirow{2}{*}{$\begin{array}{l}\text { Along the flexion/ extension axis of rotation at } \\
\text { the lateral femoral condyle. }\end{array}$} \\
\hline RKNEL & Right knee lateral & \\
\hline LKNEM & Left knee medial & \multirow{2}{*}{$\begin{array}{l}\text { Along the flexion/ extension axis of rotation at } \\
\text { the medial femoral condyle. }\end{array}$} \\
\hline RKNEM & Right knee medial & \\
\hline LCALFS & Left calf superior & \multirow{2}{*}{$\begin{array}{l}\text { Placed } 2 \text { inches below the line between the } \\
\text { L/RKNEL and the L/RKNEEM on the back of } \\
\text { the calf. }\end{array}$} \\
\hline RCALFS & Right calf superior & \\
\hline LCALFA & Left calf inferior & \multirow[t]{2}{*}{ Placed 5 inches directly below the L/RCALFS } \\
\hline RCALFA & Right calf inferior & \\
\hline LANKL & Left ankle lateral & \multirow{2}{*}{$\begin{array}{l}\text { Along the flexion/ extension axis of rotation at } \\
\text { the lateral Malleolus. }\end{array}$} \\
\hline RANKL & Right ankle lateral & \\
\hline LANKM & Left ankle medial & \multirow{2}{*}{$\begin{array}{l}\text { Along the flexion/ extension axis of rotation at } \\
\text { the medial Malleolus. }\end{array}$} \\
\hline RANKM & Right ankle medial & \\
\hline LMTPL & Left metatarsal lateral & \multirow[t]{2}{*}{ Dorsal aspect of the fifth metatarsal head. } \\
\hline RMTPL & Right metatarsal lateral & \\
\hline LMTPM & Left metatarsal medial & \multirow[t]{2}{*}{ Dorsal aspect of the first metatarsal head. } \\
\hline RMTPM & Right metatarsal medial & \\
\hline LFTC & Left foot central & \multirow{2}{*}{$\begin{array}{l}\text { Placed directly } 2 \text { inches below the lateral } \\
\text { Malleolus }\end{array}$} \\
\hline RFTC & Right foot central & \\
\hline LHL & Left heel & \multirow[t]{2}{*}{ Placed on the back of the foot. } \\
\hline RHL & Right Heel & \\
\hline
\end{tabular}

Marker set and anatomical position, with redundant markers for static capture only in bold. 